\title{
Agent-Oriented Context-Aware Platforms Supporting Communities of Practice in Health Care
}

\author{
Luiz Olavo Bonino da \\ Silva Santos \\ University of Trento \\ Via Sommerive, 14 \\ 38100 Povo, TN, Italia \\ bonino@dit.unitn.it
}

\author{
Renata S. S. Guizzardi \\ University of Twente \\ P.O. Box 1212 \\ Enschede, the Netherlands \\ souza@cs.utwente.nl
}

\author{
Marten van Sinderen \\ University of Twente \\ P.O. Box 1212 \\ Enschede, the Netherlands \\ sinderen@cs.utwente.nl
}

\begin{abstract}
This paper presents and discusses the use of an agent-oriented context-aware platform to support communities of practice (CoPs) in the health care domain. Our work is based on a scenario where CoPs are applied in a hospital to enhance the knowledge sharing among the staff members who share interests and goals. Here, we test the support of an agent-oriented modeling language (AORML) for the analysis of the proposed application for the test case scenario.
\end{abstract}

\section{Categories and Subject Descriptors}

H.4 [Information Systems Applications]: Miscellaneous

\section{General Terms}

Experimentation

\section{Keywords}

Agent-Oriented Languages, CoPs, Context-Aware Platforms

\section{INTRODUCTION}

Context-Aware computing allows software applications to use information beyond those directly provided as user input. Therefore, identical sets of user input data can produce different outputs since contextual information is considered in the processing, and context can change at each system invocation. In addition to that, this facilitates the use of these applications by minimizing user intervention, and increases their independency in gathering and processing relevant information. Here, a context is depicted by any relevant information that can be used to characterize the situation of an entity.

These features allow us to build more flexible systems to support knowledge sharing in communities of practice (CoPs) [2], which are dynamic environments, composed of people sharing interests and goals. Having this in mind, we propose the use the ContextAware Services Platform (CASP [3]) to support CoPs in the health care domain. CASP provides an infrastructure supports the use of semantically annotated web services, and it offers dynamic service discovery, dynamic deployment of new services, and context gathering from third party context providers or sensors.

COPYRIGHT IS HELD BY THE AUTHOR/OWNER(S). AAMAS'05, July 25-29, 2005, Utrecht, Netherlands ACM 1-59593-094-9/05/0007
Agents have been used as an enabling technology for organizational settings [1,2]. In our perspective, the agent paradigm is suitable i) regarding $\mathrm{CoPs}$, by providing a suitable metaphor used for modeling KM domains and systems; and ii) concerning context-aware applications, agents seem to be a natural technological approach to model and develop them, having context providers viewed as agents' sensors or other agents of the environment. But although agents seem to comply perfectly with the requirements of developing such applications, having the right abstraction is not enough for guaranteeing the development of adequate solutions. For that, consistent software engineering methods and languages are needed. In this particular paper, we test the usability of the Agent-Object-Relationship modeling language (AORML) [4].

In section 2, we present our proposed scenario; section 3 exemplifies the scenario modeling with the use of AORML; and section 4 concludes this paper.

\section{HEALTH CARE SCENARIO}

The ABC hospital sponsors CoPs development across its units. Although these communities are self-organizing groups, the hospital management fostered their formation, by providing support to their initial configuration. This has been achieved with the following measures: i) in the initial configuration, the CoPs' organization reflected the division of medical specialties; then ii) a context-aware application named Interact was deployed, supporting the emergence of new CoPs, based on interactions between members of each unit. Interact is a Client of the CASP platform, and uses an existing service named FindAFriend (FF) to find some collaborators among the hospital staff that may share interests with him/her. Interact allows the CoPs' members to fill in their profiles and interact with each other by sending e-mails, submitting comments to newsgroups, and using instant messaging. The members' profiles and the information embedded on their interactions are considered as contextual information. This information is analyzed by FF, which identifies related interests, cognitive and social characteristics for creating new CoPs.

\section{SCENARIO MODELING WITH AORML}

Figure 1 presents an AOR agent diagram. Here follows a description of each agent in the scenario:

- Hospital ABC: organization where the CoPs are developed.

- CoP: communities of practice created within and across the units of Hospital ABC. 
- Management: fosters CoPs within Hospital ABC.

- Member: the CoPs' participants and the actual users of the context-aware system. Within this class, the object Medical Specialty is the basis for the creation of the first CoPs.

- CASP Platform: context-aware services platform.

- Client: accesses services through CASP, being the actual enduser application.

- Interact: client used by Members to collaborate and receive suggestions about the creation of new CoPs.

- Context Provider: provides contextual information gathered by sensors or third party Context Providers.

- AllPersonnelCP: the Context Provider of our application.

- Service Provider: offers services by registering their description to the CASP platform.

- FindAFriend: service that uses contextual information of the Members to propose the creation of new CoPs.

A concrete example of Interact's use is the following: Ronald, a cardio-vascular surgeon exchange e-mails with Sanny, a plastic surgeon about the implications of a particular plastic surgery procedure in cardio-vascular condition. Although they are from different medical specialties, thus from different initial CoPs, they are married (social characteristic) and share common professional interests. AllPersonnelCP submits contextual information (e.g., info from exchanged e-mails, data from user's profiles) to CASP. This triggers FindaFriend to suggest the creation of a new CoP involving Ronald and Sanny. Figure 2 presents all messages exchanged between Interact and the CASP Platform, and between the CASP Platform and FindAFriend, particularly illustrating the AORML commitment construct. Note that the delivery of such recommendation to the users has been suppressed here. Basically, after the last message submitted to Interact (the SendSrvResult message), Interact suggests to all users present in LstFriends to collaborate, creating a new CoP. A commitment entitled provideSrv is automatically created between Interact and the CASP Platform when CASP acknowledges a request for a service execution has been received (AckReqSrv message). As depicted in Fig. 5, these two messages form the parameters of the provideSrv commitment, connected by an XOR diamond indicating that either of these two messages leads to the fulfillment of this commitment. Any other result consequently fails the established commitment. This commitment guarantees that CASP will either provide the requested service or, at least, send a 'service execution failed' message. This type of agreement between the agents is particularly interesting in situations like the one described here, where CASP and Interact are systems developed by different parties.

\section{CONCLUSION}

After using the AOR modeling approach in our scenario, we found it to be quite suitable to model context aware systems. First, AORML presents the advantage of modeling both active and passive entities of the scenario, differentiating between agents and objects. We found the combination of both agents and objects to be very useful, since in context-aware applications, not all entities should be represented as agents, but only those that have intentionality. The resources used by the agents, as well as agents' beliefs may be well represented as objects. Besides, AORML allows interaction modeling through three kinds of diagrams (one exemplified here), and model agent's reactive behavior through reactive rules.

\section{REFERENCES}

[1] Dignum, V. A model for organizational interaction: based on agents, founded in logic. PhD Thesis. Utrecht Univ., Jan. 2004. [2] Guizzardi, R., Perini, A., Dignum, V. Providing Knowledge Management Support to CoPs through Agent-oriented Analysis. In Proc. of 4th Int. Conf. on KM., Graz, Austria, June/2004. [3] Santos, L.O.B.S., Semantic Services Support for ContextAware Platforms, MSc Diss., UFES, Brazil, Sept., 2004.

[4] Wagner, G. The Agent-Object-Relationship Meta-Model: Towards a Unified View of State and Behavior. In Information Systems, 28:5, 2003.

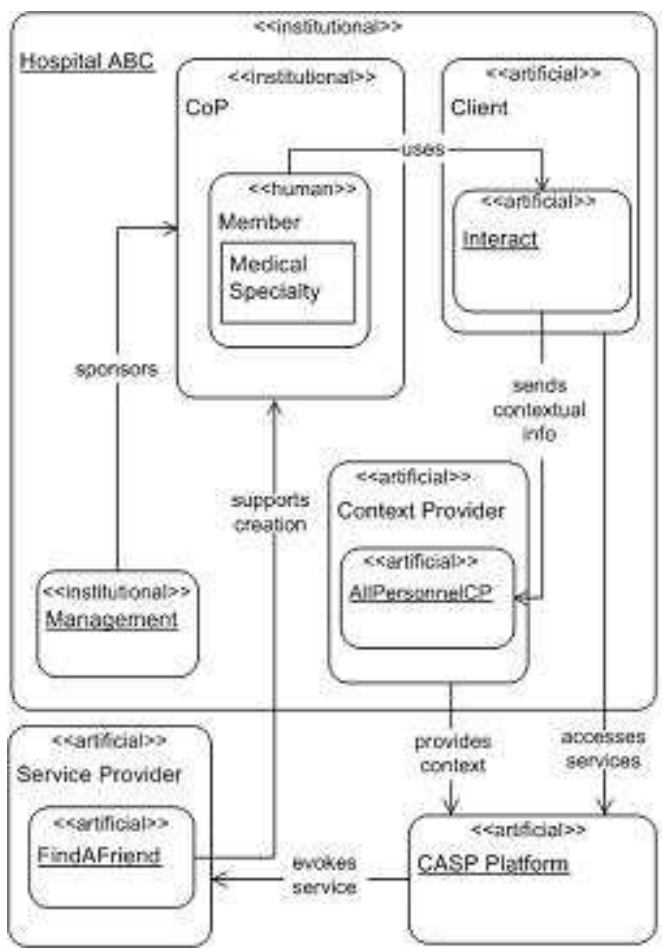

Figure 1 - Scenario modeled using the AOR Agent Diagram

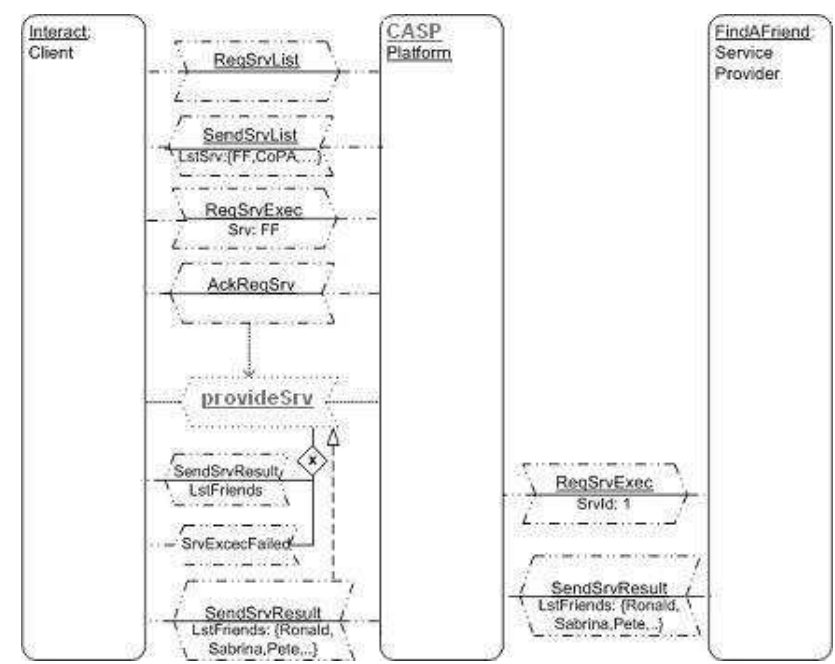

Figure 2 - AORML Interaction Sequence Diagram Showing Service Request and Execution 